Casos Clínicos

Arch. Esp. Urol., 61, 8 (939-943), 2008

\title{
METÁSTASIS NEUROLÓGICAS SECUNDARIAS A TUMOR TESTICULAR GERMINAL
}

Roberto Llarena Ibarguren, Igor Azurmendi Arín, Jorge García-Olaverri Rodríguez, Ivan Olano Grasa, Emilio Canton Aller y Carlos Pertusa Peña.

Servicio de Urología. Hospital de Cruces. Baracaldo. Vizcaya. España.

Resumen.- OBJETIVO: Las metástasis neurológicas secundarias a tumores urológicos suponen un $12 \%$ del total. Las derivadas de los tumores germinales testiculares en la época del cisplatino son excepcionales.

MÉTODOS: Presentamos un caso de tumor germinal mixto en un varón de 49 años tratado mediante quimioterapia sistémica desde 18 meses antes que presentó severa clínica neurológica central y periférica, que le condujo a la muerte por hemorragia cerebral masiva.

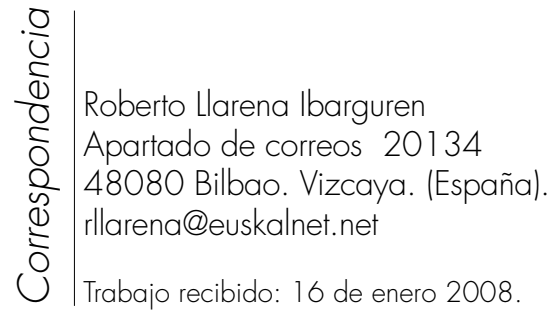


RESULTADOS: Se describen 3 tipos de presentación de las metástasis cerebrales en pacientes con cáncer testicular. El tipo 1 en el que se presentan sincrónicamente al tumor primario. El tipo 2 en el que se diagnostican tras un período de remisión luego del tratamiento citostático convencional. En el tipo 3 las metástasis se diagnostican durante el curso de la enfermedad y durante su tratamiento.

CONCLUSIONES: Salvo en caso de metástasis únicas encuadradas en el grupo 1 y 2 susceptibles de cirugía o radiocirugía, en el que cabe esperar respuesta, en el resto de lesiones secundarias a tumores germinales la evolución y el pronóstico son ominosos, con supervivencias escasas.

Palabras clave: Tumor germinal. Metástasis. Cerebro.

Summary.- OBJECTIVE: Neurological metastases secondary to urological tumors account for $12 \%$ overall. The ones derived from germ cells testicular tumors are exceptional in the age of cisplatin.

METHODS: We report one case of mixed germ cell tumor in a 49-year-old male patient treated with systemic chemotherapy during 18 months before presenting with severe central and peripheral neurological symptoms leading to death due to massive cerebral hemorrhage.

RESULTS: We describe three types of presentation of cerebral metastases in patients with testicular cancer. Type 1 present synchronically with the primary tumor. Type 2 are diagnosed after a period of remission after conventional cytostatic treatment. Type 3 metastases are diagnosed during the course of the disease and its treatment.

CONCLUSIONS: Except unique metastases classified in groups 1 and 2, which are susceptible of surgery or radiosurgery, in which in response may be expected; the rest of lesions secondary to germ cell tumors have an ominous prognosis and outcomes, with short survivals.

Keywords: Germ cell tumor. Metastasis. Brain.

\section{INTRODUCCIÓN}

Las metástasis cerebrales de origen urológico son una entidad infrecuente y poco referenciada en nuestro medio.

Sabemos que el $50 \%$ de los tumores cerebrales son secundarios y que entre el 24 y el $45 \%$, dependiendo de trabajos, de pacientes fallecidos por cáncer presentan diseminación cerebral (1).
Nussbaum (2) en 1996 estableció los porcentajes achacables a cada órgano responsable de la diseminación neurológica. Así el pulmón era el órgano primario que con más frecuencia, $40 \%$, originaba metástasis cerebrales, seguido con un $17 \%$ de la mama, y con un $11 \%$ el melanoma. El mismo autor publicó basándose en un grupo de 729 pacientes que el origen urológico global era del $12 \%$, dividiéndolo en un $6 \%$ para el riñón, seguido a partes iguales del $2 \%$ por la vejiga, la próstata y el testículo. De esta manera podemos darnos cuenta de la importancia que tiene nuestra patología tumoral.

Existen autores como Mahalatti (3) que nos relata como antes del advenimiento del cisplatino como droga básica y fundamental en el control del tumor testicular, hasta el $40 \%$ de los pacientes que morían por el tumor lo hacían con metástasis cerebrales, mientras que en la actualidad tan solo ocurre esta situación en el $2 \%$.

\section{CASO CLÍNICO}

Presentamos el caso de un varón diagnosticado a los 49 años de un fumor testicular germinal derecho de estirpe mixta que incluía un predominio de carcinoma embrionario junto a focos de seno endodérmico. Se estadió como $\mathrm{pT} 1 \mathrm{~N} 3 \mathrm{M} 1 \mathrm{~b}$ (L3) S1 con positividad tanto para alfafetoproteína como para la $\beta$ coriogonadotrofina. Se administrarón 4 ciclos sucesivos de cisplatino y etopósido, logrando negativización de los marcadores tumorales tras el segundo ciclo, y obteniendo respuesta parcial pulmonar, junto a estabilización de la enfermedad retroperitoneal. No se planteó cirugía de masa retroperitoneal dada la presencia de lesiones pulmonares.

Ocho meses después se reproducen las lesiones pulmonares, elevándose los marcadores germinales. Se trata mediante 4 ciclos de citostáticos que incluyeron cisplatino, etopósido e ifosfamida, obteniéndose remisión parcial mensurable tanto de las lesiones pulmonares como de las retroperitoneales, así como una negativización temprana de los marcadores tumorales.

Cinco meses después se evidencia una progresión ganglionar mediastínica, sin progresión de las lesiones previas ni de los marcadores tumorales. Se inician y completan 4 nuevos ciclos de cisplatino, etopósido e ifosfamida, obteniéndose remisión parcial y objetiva de la diseminación ganglionar torácica.

Pasados tan solo 3 meses de la finalización del último bloque de cistostáticos ingresa de urgencia por paresia de ambas extremidades inferiores, junto a cefalea frontal y obnubilación. Se realiza estudio neurológico mediante resonancia magnética nuclear que pone de manifiesto la existencia de lesión sólida en hemisferio izquierdo, región temporoparietal, con amplia área hemorrágica (Figura 1), además de 3 lesiones sólidas intradurales a nivel C7-D 1, L2-L3 y L5-S1 (Figuras 2 y 3). 


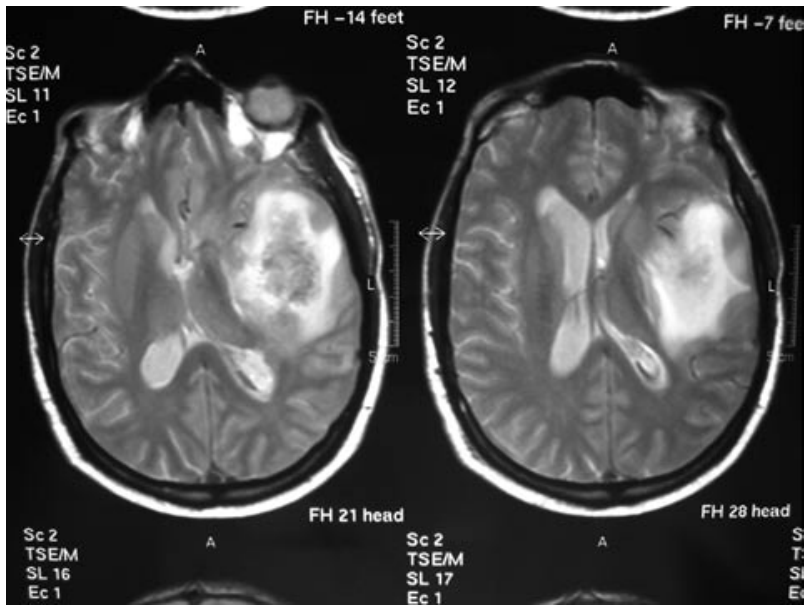

FIGURA 1. RMN. Cortes axiales donde se aprecia lesión localizada en región temporoparietal izquierda que presenta gran componente hemorrágico.

Una vez instauradas medidas antiedema con corticoides a altas dosis y manitol, se procede a reinicio de quimioterapia con cisplatino, ifosfamida y taxoles, tras lo que la situación del paciente empeoró por nuevo resangrado cerebral falleciendo 15 días después.

\section{COMENTARIOS}

Aunque la mayoría de las publicaciones se refieren a 1 - 2 pacientes como mucho, existen trabajos que presentan muchos casos de metástasis cerebrales secundarias a tumores testiculares.
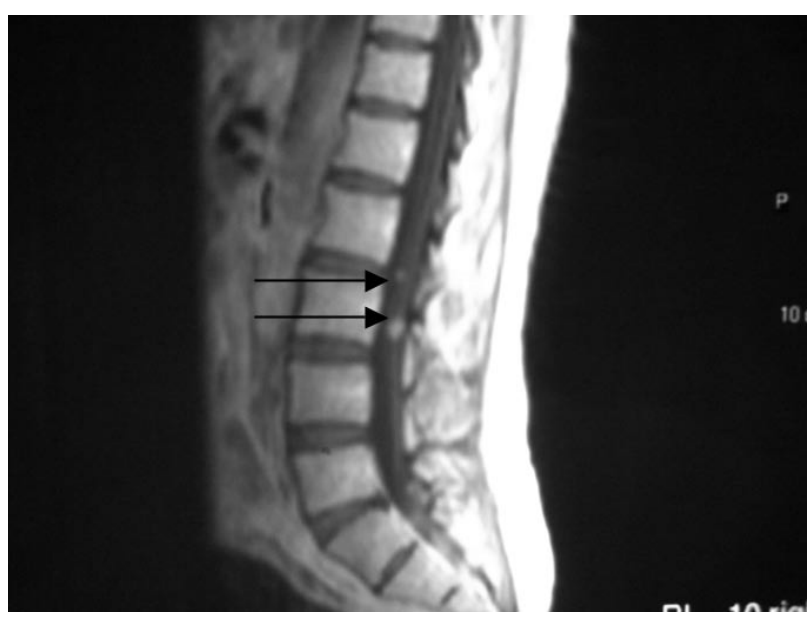

FIGURA 2. RMN. Corte sagital en el que se aprecian lesiones intradurales a nivel L2-L3.
La mayor serie, del año 97, se debe a Bokemeyer (4) que presenta 44 casos, de los que 42 fueron secundarios a tumores no seminomatosos, siendo tan solo responsable de 2, el seminoma. Cleamm en 1993 (5) publicó una serie compuesta de 29 casos, de los que 27 eran no seminomatosos y 2 seminomas. Spears (6) en 1992, del grupo de la Universidad de Indiana publicó 24 casos. Previamente y en el año 1981 Higi referenció 16 casos (7) en una serie de 314 pacientes. Más recientemente, Crabb en 2002 (8) publica 15 casos pero sobre un total de 1049 tumores testiculares. Con 11 casos encontramos a Mahalatti en 1999 (3) y a Lutterbach (9) encontradas sobre un total de 914 lesiones metastásicas cerebrales sometidas a radioterapia. Con menor número de casos tenemos a Rustin (10) con 9, Gerl (11) con 6 de un total de 416 tumores y Raina (12) con 3 metástasis de un total de 123 casos.

El primer mensaje que podemos obtener analizando las series previas es que la mayoría de los tumores testiculares que desarrollan metástasis neurológicas son de estirpe no seminomatosa. El segundo, que el número de metástasis cerebrales en la época del cisplatino es considerablemente más baja que cuando no se disponía de él como droga básica.

La distribución topográfica de las metástasis refleja la irrigación sanguínea cerebral. Así las células anidan en la zona de unión de la sustancia blanca con la gris, irrigada por arterias superficiales distales (13). A pesar de que el cerebro es asiento del $80 \%$ de metástasis, no hay que olvidar que el $15 \%$ restante asentarán en cerebelo y el otro $5 \%$ en medula espinal (1). Curiosamente los tumores pelvianos, de útero o próstata tienen predilección por la fosa posterior hasta en un $50 \%$ de los casos, situación que tan solo se produce en el $10 \%$ del resto de tumores (1).

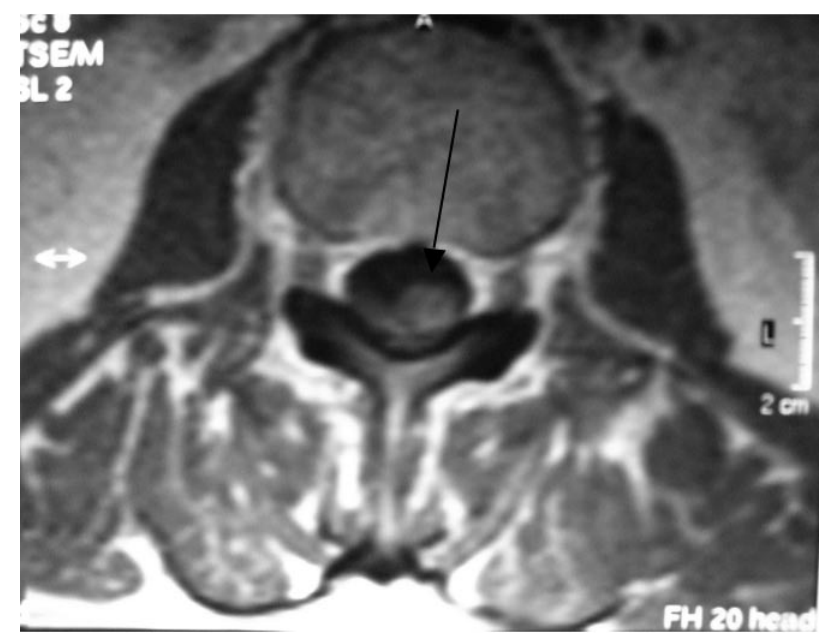

FIGURA 3. RMN. Corte axial a nivel 13 Lesion intradural. 
Respecto al número de metástasis, en el estudio de Nussbaum (2) el $53 \%$ de los casos presentaban lesiones únicas, mientras que en el $47 \%$ las lesiones eran más de dos. De cara a un planteamiento terapéutico es interesante la clasificación que ofrece Ewend (14) en cuanto al número de metástasis en únicas o solitarias, oligometástasis, cuando son 2 ó 3 , y múltiples cuando su número es de 4 o más.

Clínicamente pueden presentarse con cefalea intensa intratable, vómitos severos, hemiparesia, alteraciones cognitivas, ataxia, alucinaciones, convulsiones hasta en el $20 \%$ de los casos y demás síntomas neurológicos provocados por las lesiones ocupantes de espacio (3). Lógicamente la clínica variará en relación a la localización de la lesión, y al número de éstas (13).

Aunque por la clínica la sospecha sea alta, estaremos obligados a certificar la existencia de metástasis neurológicas. En la actualidad tanto la TAC como la RMN son los métodos más seguros y eficaces a la hora del diagnóstico $(13,15)$.

Dado que Brem (13) nos apunta la posibilidad de que hasta un $10 \%$ de las metástasis sean asintomáticas, unido a la evidencia de que casi considerada como condición previa a la aparición de metástasis cerebrales sea la existencia de amplia enfermedad torácica $(3,4,11)$ y de que hasta el $32 \%$ de los casos con afectación pulmonar y mediastínica presentan diseminación cerebral (4) se impone en estos casos de enfermedad supradiafragmática masiva el estudio cerebral mediante TAC a fin de descartar metástasis asintomáticas, que pudieran llegar a ser incluso quirúrgicas (3).

En 1992, Spears (8) del grupo de Indiana clasifica en 3 grupos los casos de metástasis cerebrales en relación al tiempo de aparición o diagnóstico de las mismas. Así el grupo 1 serían los casos de diagnóstico al inicio del tratamiento, el grupo 2 cuando aparecen tras un período de remisión luego del tratamiento quimioterápico, y el grupo 3 en los que aparecerán durante el curso del tratamiento. De esta clasificación se derivan implicaciones pronósticas en relación al estado de quimiosensibilidad, considerándose quimiosensibles las del grupo 1 , debiendo de cambiar la estrategia terapéutica en el grupo 2 buscando quimiosensibilidad, y estableciendo una situación de quimioinsensibilidad o resistencia en el grupo 3 (4).

De esta ordenación en grupos se vuelve al concepto de "santuario cerebral", situación propiciada por la existencia de la barrera hematoencefálica que "protegería" a la lesión cerebral existente de la acción de los citostáticos, sobre todo en el grupo 2 , en los que las lesiones debutarían tras remisiones de las metástasis en otros lugares de la economía $(6,8)$.

En la serie de Bokemeyer (4) compuesta de 44 casos, el $41 \%$ de ellos se encuadraron en el grupo 1 , y el $50 \%$ en el grupo 3, siendo tan solo el $9 \%$ de los pacientes del grupo 2. Lutterbach (9) presentó 11 casos, 2 del grupo 1,3 del grupo 2, y 6 del grupo 3 . Spears (6) publica 24 pacientes, 10 del grupo 1, 4 del grupo 2, y 10 del grupo 3. Como puede apreciarse, aproximadamente el $40 \%$ de los pacientes se encuadran en el grupo 1, diagnosticadas al inicio, casi el $50 \%$ en el grupo 3 , enfermedad progresiva durante el tratamiento quimioterápico, y alrededor del $10 \%$ en el grupo 2, aparecidas luego de la remisión del resto de las lesiones.

El tratamiento deberá ser individualizado, ya que por la escasa experiencia de cada grupo, la terapia no está lo suficientemente protocolizada. Además la orientación terapéutica deberá estar en consonancia con los demás factores pronósticos.

Distinguiremos las metástasis asintomáticas, escasas, de las sintomáticas. En éstas, en primer lugar habrá que estabilizar al paciente, mediante corticoides a altas dosis, de preferencia empleando dexametasona con dosis de hasta $40 \mathrm{mg}$ diarios como choque a fin de controlar el edema (14). También serán necesarios el manitol, y los anticomiciales frente a las convulsiones (14). En pacientes con grave hipertensión intracraneal se considerará la derivación ventricular.

Una vez estabilizado al paciente se deberá estadiar la situación neurológica, relacionándola y encuadrándola en uno de los 3 grupos pronósticos. En el grupo 1 la indicación terapéutica será la quimioterapia basada en cisplatino y la radioterapia $(6,14)$. En el grupo 2 , la quimioterapia de segunda línea, y la radioterapia, valorando la cirugía en casos de metástasis únicas, menores de $2 \mathrm{~cm}(2,14)$. En el grupo 3, tan solo se contempla la radioterapia (6).

Hay autores (4) que plantean la quimioterapia inicial agresiva con esquemas POMB-ACE o VIP, en todos los grupos. Asimismo proponen la cirugía de las lesiones únicas luego de la inducción citostática, reservando la radioterapia con dosis de hasta 50 cGy para las metástasis múltiples y en las que existan células viables luego de la exéresis. A la vez contraindican la cirugía en lesiones múltiples o en aquellas del grupo 3, aparecidas durante el tratamiento quimioterápico.

Apoyándose en la contundencia obligada como norma en el tratamiento de las metástasis cerebrales de origen germinal, Ewend (14), propone la cirugía, la radiocirugía o la radioterapia con 30cGy en lesiones únicas e inferiores a $3 \mathrm{~cm}$., pero aconsejando la cirugía siempre que se considere el tumor primario como quimiosensible, situación propicia en tumores de mama, de célula pequeña pulmonar y testiculares. En casos de existir 2 - 3 lesiones, apoya el uso de radioterapia con 30 cGy divididas en 10 sesiones administradas a lo largo de 10 días. El mismo autor aboga por volver a emplear la radiocirugía, incluso, pese al riesgo de radionecrosis, la radioterapia en los casos de recurrencia de la enfer- 
medad, sobre todo cuando han presentado respuesta objetiva al primer tratamiento.

Para dar la impresión del mal pronóstico y pobres respuestas terapéuticas obtenidas con los diferentes tratamientos, existen publicados remedios excepcionales como la asociación de hipertermia (16) hasta de $41^{\prime} 8^{\circ} \mathrm{C}$, logrando efecto tumoricida, relatando buenas supervivencias de hasta 5 años.

El pronóstico en general es malo. A pesar de todas las medidas sugeridas por Ewend (14) y de conseguir un $42 \%$ de respuestas, la supervivencia ronda los 5 meses de media. Todos los autores coinciden en el ominoso pronóstico que presentan los pacientes del grupo 3 . En general se considera una supervivencia media de 6 meses estudiando todas las series (9). En los casos de los grupos 1 y 2 .ocasionalmente se han comunicado supervivencias de hasta 90 meses (5) e incluso de 194 meses (9), que debiéramos considerarlas anecdóticas.

\section{CONCLUSIONES}

Las metástasis neurológicas secundarias a tumores germinales de testículo son raras. A pesar de haberse publicado lesiones de asiento medular, las cerebrales son las más frecuentes. La mayoría aparecen en pacientes con enfermedad metastásica torácica de gran volumen, por lo que se recomienda la realización de medios diagnósticos de cara a descartarlas en estos casos. Se dividen en grupos según su aparición en relación al diagnóstico del tumor primario. En el grupo 1 las metástasis son sincrónicas al tumor testicular. En el grupo 2 aparecen después de la remisión del resto de lesiones. En el grupo 3 , de peor pronóstico, la diseminación ocurre durante el tratamiento sistémico del tumor. El tratamiento deberá individualizarse dependiendo del grupo al que asignemos al paciente, y del número, tamaño y localización de las lesiones. La base terapeútica se fundamenta en quimioterapia sistémica, radioterapia y radiocirugía, y cirugía en lesiones únicas menores de $2 \mathrm{~cm}$.

\section{BIBLIOGRAFÍA y LECTURAS RECOMENDADAS (*lectura de interés $y^{* *}$ lectura fundamental)}

*1. POSNER, J.B.; CHERNIK, N.L.: "Intracraneal metastases from systemic cancer". Adv. Neurol., 19: 579, 1978.

*2. NUSSBAUM, E.S.; DJALILIAN, H.R.; CHO, K.H. y cols.: "Brain metastases: Histology, multiplicity, surgery and survival". Cancer, 78: 1781, 1996.
**3. MAHALATI, K.; BILEN, C.Y.; ÖZEN, H. y cols.: "The management of brain metastasis in nonseminomatous germ cell tumours". BJU International, 83, 4: 457, 1999.

*4. BOKEMEYER, C.; NOVACK, P., HAUPT, A. y cols.: "Treatment of brain metastases in patients with testicular cancer". Journal of Clinical Oncology, 4: 1449, 1997.

5. CLEMM, C.; GERL, A.; WENDT, T.G. y cols.: "Current status of therapy of CNS metastases of germ cell tumors". Urologe, 3: 217, 1993.

6. SPEARS, W.T.; MORPHIS, J.G.; LESTER, S.G. y cols.: "Brain metastases and testicular tumors: Long term survival". International Journal of Radiation Oncology, Biology, Physics. 1: 17, 1992.

7. HIGI, M.; SCHEULEN, M.E.; SCHMODT, C.G. y cols.: "Brain metastases in malignant testicular teratomas". Onkologie, 2: 84, 1981.

8. CRABB, S.J.; McKENDRICK, J.J.; MEAD, G.M.: "Brain as sanctuary site of relapse in germ cell cancer patients previously treated with chemotherapy". Clinical Oncology, 4: 287, 2002.

9. LUTTERBACH, J.; SPETZGER, U.; BARTELT, S. y cols.: "Malignant germ cell tumores metastatic to the brain: A model for a curable neoplasm? The Freiburg experience and a review of the literature". Journal of Neuro-Oncology, 2: 147, 2002.

10. RUSTIN, G.J.; NEWLANDS, E.S.; BAGSHAWE, K.D.: "Succesful management of metastatic and primary germ cell tumors in the brain". Cancer, 11: 2108, 1986.

11. GERL, A.; CLEMM, C.; KOHL, P. y cols.: "Central nervous systems sanctuary site replapse in patients treated with chemotherapy for metastatic testicular cancer". Clinical \& Esperimental metastasis, 3: 226, 1994.

12. RAINA, V.; SINGH, S.P.; KAMBLE, N. y cols.: "Brain metastases as the site of relapse in germ cell tumour of testis". Cancer, 7: 2182, 1993.

13. BREM, S.; PANATTI, J.G.: "An era of rapid advancement; Diagnosis and treatment of metastatic brain cancer". Congress of neurological surgeons, 57: 5, 2005.

**14. EWEND, M.; ELBABAA, S.; CAREY, L.A.: "Current treatment paradigms for the management of patients with brain metastases". Neurosurgery, 5: 75, 2005.

15. MIRANDA, P.; RAMOS, A.; RICOY, J.R.: "Images in neuro-oncology: Brain metastases and leptomeningeal dissemination of nonseminomatous germ cell tumor". Journal of Neuro-Oncology, 73: 51, 2005.

16. LALLAVE, F.; LOMAS, M.; LAGUNA, E. y cols.: "Estudio descriptivo de los tumores testiculares germinales: 13 años de experiencia en el área de salud de Badajoz". Arch. Esp. Urol., 60: 531, 2007.

17. FEVERABEND, T.; WIEDEMANN, G.J.; STEEVES, R.: "Advanced non-seminomatous germ cell cancer of the testis with brain metastases: Feability of additional brain irradiation and whole body hyperthermia plus chemotherapy". Oncology reports, 2: 219, 2001. 